\title{
RADIATIVE CORRECTIONS TO VECTOR BOSON MASSES FOR HEAVY HIGGS BOSONS
}

\author{
Martin B. EINHORN \\ NORDITA, DK-2100 Copenhagen Ø, Denmark \\ and Randall Laboratory of Physics, University of Michigan, Ann Arbor, MI 48109, USA \\ and \\ Hajime KATSUMATA \\ Randall Laboratory of Physics, University of Michigan, Ann Arbor, MI 48109, USA
}

Received 16 August 1986

Radiative corrections to the masses $M$ of the vector bosons $\mathrm{W}$ and $\mathrm{Z}$ increase in perturbation theory with increasing Higgs mass $m$. Since these masses will be experimentally determined to an accuracy on the order of $0.1 \%$, these measurements may offer one of the best tests of the detailed dynamics of the standard model. In particular, they would seem to place an upper limit on the Higgs mass $m$ (or, since $m^{2} \sim \lambda$, the maximum strength of the scalar self-coupling $\lambda$ ). Performing a $1 / N$ expansion in $\mathrm{SU}(N) \times \mathrm{U}(1)$ through order $1 / N$ but all orders in the scalar coupling $\lambda N$, it is demonstrated that the radiative corrections $\delta M^{2}$ to the vector boson masses are of the form $\delta M^{2} / M^{2}=\left(g^{2} N / 16 \pi^{2}\right)\left[F_{1}\left(\theta_{\mathrm{W}}\right)+N^{-1} F_{2}\left(\lambda N, \theta_{\mathrm{W}}\right)\right.$ $+\mathrm{O}\left(N^{-2}\right)$ ], where $g^{2} N$ is the $\mathrm{SU}(N)$ gauge coupling constant and $\theta_{\mathrm{w}}$ the weak mixing angle. The function $F_{2}$ remains finite even in the limit of infinite self-coupling constant. Thus, no matter how large the perturbative Higgs mass $m$ is, the vector boson mass shifts may remain small, on the order of the anticipated experimental accuracy.

The most mysterious aspect of the standard model by far concerns the role of the scalar Higgs field and its quantum, the Higgs boson. As yet, its only manifestation is in the masses of the $W$ and $Z$ vector bosons, and the experimental bounds on its mass are meager. Certain aesthetic and theoretical considerations such as naturalness [1] and the triviality of scalar field theory [2] lead us to regard the Higgs sector as a phenomenological or effective field theory below some energy scale $\Lambda_{\mathrm{c}}$. Naturalness arguments suggest that this scale $\Lambda_{\mathrm{c}}$ cannot be very far above the weak scale $v \sim 250$ $\mathrm{GeV}$. It may well be that as the scale of the cutoff is approached, the scalar self-coupling $\lambda(\mu)$ becomes large. This strong interaction may also be motivated by a desire to make the Higgs mass so heavy as to drive it out of the theory [3]. However, although the perturbative Higgs mass $m \equiv \sqrt{2 \lambda} v$ increases with $\lambda$ (for fixed $v$ ), it is an open question what happens if $\lambda$ becomes so large that the perturbation expansion is no longer a reliable way to estimate its effects.

Two methods have been introduced to study this strong coupling limit: (a) numerical simulation on a lattice ${ }^{\neq 1}$, and (b) an expansion in the inverse number of degrees of freedom, called a $1 / N$ expansion $[5,6]$. Both approaches suggest the same conclusion: within the standard model, there is a finite limit to how large the physical Higgs mass can be. This upper limit is fixed by the scale of the cutoff $\Lambda_{c}$ required by triviality, beyond which predictions of the local scalar field theory cannot be trusted [5].

One is led to wonder whether other radiative corrections will remain small when $\lambda$ becomes large. In particular, the masses of the vector bosons [7] agree well with their perturbatively estimated values. These masses will be measured in future experiments to high

\$1 A review of recent lattice calculations can be found in ref. [4]. 
accuracy, on the order of a few tenths of a percent. Consequently, they may serve as careful checks on the underlying field theory ${ }^{\neq 2}$ and, one might think, provide some restriction on the magnitude of $\lambda$ inasmuch as the perturbative radiative corrections are expected to grow as increasingly high powers of $\lambda$. Perturbatively, up to logarithmic factors, the Higgs mass is known to be independent of $\lambda$ at one loop [3] and proportional to $\lambda$ at two loops [9]. We conjecture that, in $n$ loops, the perturbative contribution grows as $\lambda^{n-1}$.

These considerations motivated us to attempt to calculate these corrections via a $1 / N$ expansion in an effort to get some insight beyond perturbation theory. As before [5], we consider the SU(N) $\times \mathrm{U}(1)$ model in the limit $N \rightarrow \infty$ for fixed values of $\lambda N, g^{2} N$, and $g^{\prime 2} N$, where $g$ and $g^{\prime}$ are the $\mathrm{SU}(N)$ and $\mathrm{U}(1)$ gauge couplings, respectively. We find that the form of the radiative corrections to the vector boson mass $M$ may be written as

$$
\begin{aligned}
& \delta M^{2} / M^{2}=\left(g^{2} N / 16 \pi^{2}\right) \\
& \quad \times\left[F_{1}\left(\theta_{\mathrm{w}}\right)+N^{-1} F_{2}\left(\lambda N, \theta_{\mathrm{w}}\right)+\mathrm{O}\left(N^{-2}\right)\right],
\end{aligned}
$$

where we have expanded to lowest non-trivial order in the gauge couplings, but summed to all orders in the self-coupling $\lambda N$. Here, the weak mixing angle $\theta_{\mathrm{w}}$ is conveniently defined by

$$
\tan ^{2} \theta_{\mathrm{w}}=\frac{N}{2(N-1)} \frac{g^{\prime 2} N}{g^{2} N} \rightarrow \frac{g^{\prime 2} N}{2 g^{2} N} \quad(N \rightarrow \infty) .
$$

The $\mathrm{O}(1)$ correction $F_{1}\left(\theta_{\mathrm{w}}\right)$ is completely independent of the self-coupling $\lambda N$, while the term of $\mathrm{O}(1 / N)$ depends on it. However, one can show that, in the limit $\lambda N \rightarrow \infty, F_{2}\left(\lambda N, \theta_{\mathrm{w}}\right)$ tends to a finite limit, a result we will refer to as saturation.

Since the actual calculation is rather complicated, we can only indicate, in this note, how the calculation was carried out an how certain technical hurdles were overcome. The details will be given elsewhere [10]. The general structure of the $\mathrm{SU}(N) \times \mathrm{U}(1)_{Y}$ model has been given previously [5] to which we refer the reader for notation. Unlike before, the gauge couplings cannot be neglected. The Higgs multiplet is taken to be in the fundamental representation of $\mathrm{SU}(N)$ with

\footnotetext{
\$2 Perturbative calculations have been reviewed in ref. [8].
}

$\phi \equiv\left|\begin{array}{c}\mathrm{iS}_{1} \\ \text { iS }_{2} \\ \vdots \\ \text { S }_{N-1} \\ (1 / \sqrt{2})(\sigma+v-\mathrm{i \chi})\end{array}\right|$,

where $v \equiv \sqrt{\mu} / \lambda$ is its vacuum expectation value in tree approximation. After spontaneous sy mmetry breaking, the particles are classified according to the unbroken $\mathrm{SU}(N-1) \times \mathrm{U}(1)_{Q}$ symmetry. The calculation is most conveniently performed in the Landau gauge, where the propagators have the simple forms displayed in fig. 1 . There are $N-1$ charged $\mathrm{W}$ bosons, one neutral $\mathrm{Z}$, and one photon $\mathrm{A}$. In addition, there are $(N-1)^{2}-1=N(N-2)$ massless vector bosons $B$ which do not exist for the standard model where

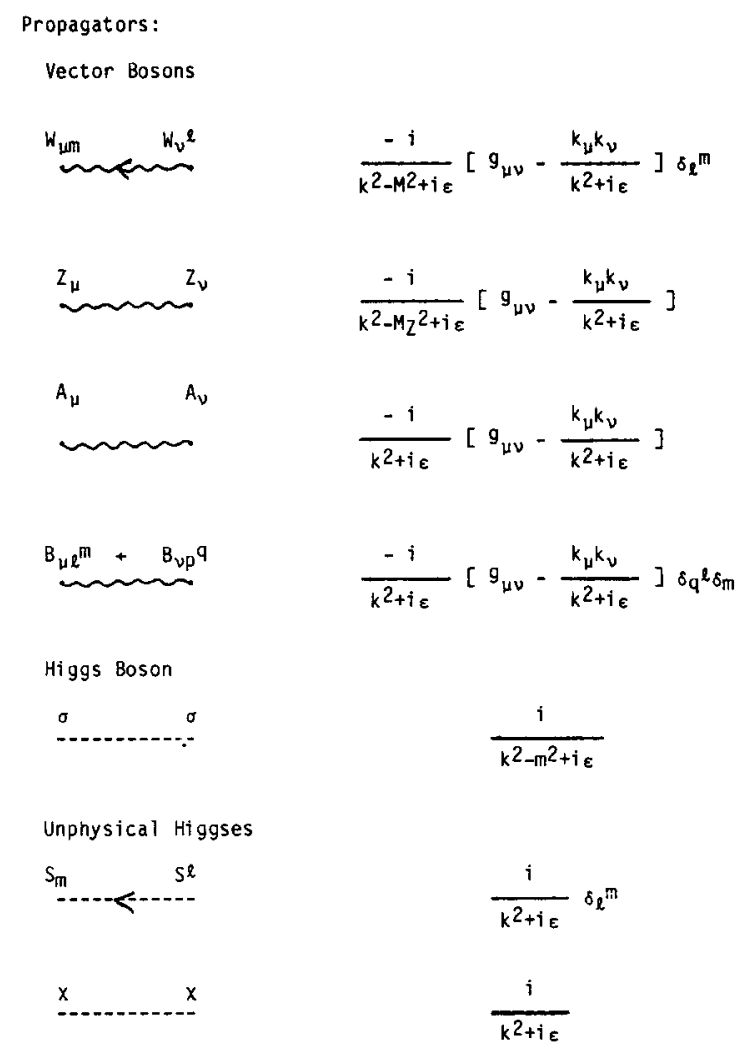

Fig. 1. Propagators in the Landau gauge. Here $\mu, \nu$ denote space-time indices; $l, m$, SU(N-1) indices. 
$N=2^{\ddagger 3}$. There is the Higgs scalar of mass $m$, and the neutral unphysical Higgs $\chi$, and the $N-1$ charged Higgses $\mathrm{S}^{-}$. Finally, not depicted in fig. 1 , there are Faddeev-Popov ghosts associated with each vector, in Landau gauge; the vector propagators are transverse and all unphysical quanta (unphysical Higgses and Faddeev-Popov ghosts) are massless, a property which engenders considerable simplification and transparency to the calculation. To maintain gauge invariance of the regulator, we employ dimensional regularization; however, we do not renormalize graph-by-graph as one does perturbatively but rather we determine counterterms after summing to all orders in $\lambda N$.

To calculate the vector boson mass shifts, one must calculate the polarization tensors for $\mathrm{W}$ and $\mathrm{Z}$. Owing to certain Ward-Takahishi identities [11], the polarization tensor is transverse,

$\Pi_{\mu \nu}\left(k^{2}\right)=\Pi\left(k^{2}\right)\left(g_{\mu \nu}-k_{\mu} k_{\nu} / k^{2}\right)$.

Because we are expanding to lowest (non-trivial) order in the gauge couplings, it suffices to evaluate the polarization tensor $\Pi_{\mu \nu}\left(k^{2}\right)$ at the tree values $k^{2}=M^{2}$, or $k^{2}=M_{Z}^{2}$.

However, because the vector boson masses are themselves proportional to the gauge coupling, a graph may have a nontrivial and non-analytic dependence on $M^{2} / m^{2} \sim g^{2} / \lambda$. As a result, the classification by powers of $g^{2}$ and $\lambda$ may differ, for example, depending on whether $g^{2} \gg \lambda$ or vice versa. Dependence to the external momentum $k^{2}$ may also introduce factors of $g^{2}$ when $k^{2}$ is put on the mass shell since $M^{2}=\frac{1}{4} g^{2} v^{2}$. As a result, a certain amount of care must go into the classification of diagrams in the spontaneously broken phase. We found it, furthermore, convenient to evaluate $\Pi\left(k^{2}\right)$ by calculating only terms contributing to $\Pi_{\mu \nu}$ proportional to $k_{\mu} k_{\nu}$. Consider first the contributions of $O(1)$. Among the quantities one needs to include are the $\mathrm{O}(1)$ corrections to the unphysical Higgs self-energy $\Sigma_{S}$. However, in Landau gauge, these are just the same as the Goldstone bosons for the $\mathrm{O}(2 N)$ non-gauge theory,

\$3 One may legitimately worry that there are of order $N^{2}$ vector bosons which do not exist in the standard model. However, as will be seen below, our results depend upon the saturation of radiative corrections to the unphysical Higgs bosons $S$ and not upon intricate cancellations involving these vectors. with self-energies given by diagrams resembling beavertail cacti [5]. But, in dimensional regularization, a massless tadpole is zero, so the $\mathrm{O}(1)$ corrections to $\Sigma_{\mathrm{S}}$ vanish, one of the advantages of the Landau gauge. No other contributions of this order in $1 / N$ involve the self-coupling $\lambda N$ or the Higgs mass, so we have established the form of the first term in eq. (1) ${ }^{\neq 4}$. Moreover, since the graphs have the structure of one- or two-loop diagrams, it is plausible that the function $F_{1}$ is of order 1 in magnitude (and not of order, say, $16 \pi^{2}$ ).

Now we turn to the heart of the calculation, the $\mathrm{O}(1 / N)$ corrections which depend non-trivially on $\lambda N$. Unfortunately, there are a great many diagrams whose discussion quickly becomes a bit lengthy. Here, we will simply outline the ingredients. Diagrams involving the Higgs itself are already of $\mathrm{O}(1 / N)$, so we need only the $O(1)$ Higgs self-energy previously determined $[5,6]$. Recall that this has a finite non-zero limit if we formally take $\lambda N \rightarrow \infty$. However, we also need the $\mathrm{O}(1 / N)$ contributions to $\Sigma_{\mathrm{S}}$ (for zero gauge couplings) which can also be calculated and shown to saturate as $\lambda N \rightarrow \infty$. The $\mathrm{W}$ polarization tensor involves a large number of diagrams which could potentially contribute to a $k^{\mu} k^{\nu}$ term, a subset of which are depicted in fig. 2. Other diagrams involve either the exchanges of vertices or $\mathrm{O}(1)$ propagator or vertex insertions along with appropriate counterterms for divergences associated with the diagrams or subdiagrams. The diagrams of order $\left(g^{2} N\right)^{2}$ would appear to be necessary in case the Feynman integrals should yield contributions proportional to $v^{2}$. In fact, they are proportional to $k^{2}=M^{2}$ and can be discarded since this then is of higher order in $\left(g^{2} N\right)$.

Putting everything altogether, we obtain a contribution of the form of the second term in eq. (1). Remarkably enough, the function $F_{2}(\lambda n)$ can be shown to saturate, i.e., approach a finite number. Although the second term is zero for $N \rightarrow \infty$, it is inviting to try to estimate its magnitude for $N=2$ to get some indication of the generic size of the correction. The

${ }^{\ddagger 4}$ Of course, since each term in the $1 / N$ expansion is separate. ly gauge invariant, this result could be established in any gauge. However, it is extremely convenient in going beyond leading order in $1 / N$ to have the leading order selfenergy $\Sigma_{S}$ vanish and, thereby, having the independence of $\lambda N$ manifested graph-by graph. 

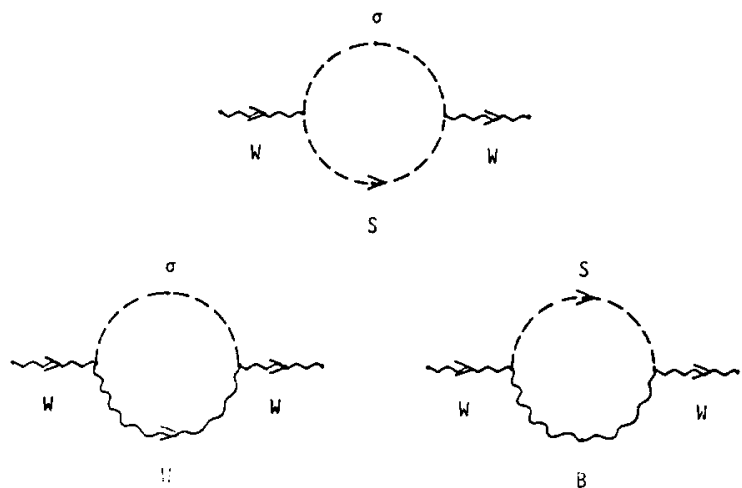

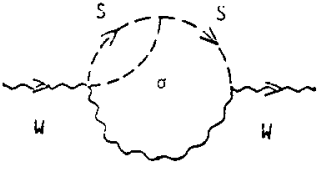

B.

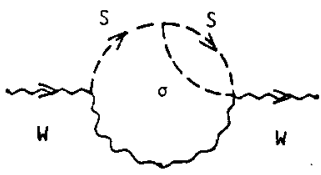

8

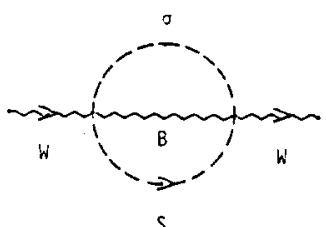

$S$

Fig. 2. Some primitive diagrams of $O(1 / N)$.

generic form of the sum of all contributions looks like one-loop integrals with modified propagators and vertices which, however, saturate in the limit $\lambda N \rightarrow \infty$. Thus, we would guess that $F_{2}$ tends to a number of order 1 for the same reason as our estimate of $F_{1}$. This leads us to suspect that the maximal magnitude of the radiative corrections is still quite small and insensitive to the Higgs self-coupling no matter how large it may become.

There may be a simple intuitive way to understand the origin of these results. Given the renormalized coupling $\lambda N(\mu)$ on some scale $\mu$, there is a finite momentum $\Lambda_{\mathrm{c}}$ (the cutoff) at which the coupling goes to infinity (triviality). The coupling constant may be written as

$\lambda N / 4 \pi^{2}=1 / \ln \left(\Lambda_{\mathrm{c}} / \mu\right)$,

so that any scale $\mu<\Lambda_{\mathrm{c}}$, there is a finite upper limit to the strength of the self-coupling. One may think of scanning different theories by varying $\Lambda_{c}$. The results of ref. [5] may be viewed as follows. The Higgs mass $m$ is of order $m^{2} \approx 2 \lambda N\left(m^{2}\right) v^{2}$. As the cutoff $\Lambda_{\mathrm{c}}$ is lowered toward $v$, the Higgs mass increases un- til there comes a point where it begins to exceed the cutoff. Clearly a local field theory approximation to the underlying dynamics makes no sense beyond this point, so one can conclude that either the Higgs excitation is not present below this lowest value of the cutoff on the effective, low-energy, local field theory or else it has a mass less than this minimum possible value of the cutoff (of order $4 \pi \sqrt{v^{2} / N}$ ). It is in this sense that there is an upper limit to the Higgs mass. Now at any energy scale $\mu$ below this cutoff $\Lambda_{c}$, the self-coupling has a finite value (given by eq. (5) in the $1 / N$ approximation) which decreases with decreasing scale. In particular, on the scale of the vector boson masses, the self-coupling may be within the range for which perturbation theory provides a reliable estimate of the magnitude of radiative corrections. As a result, the one-loop shifts in the vector bosons' masses are reliable, provided the self-coupling is given its value on the scale of vector boson masses.

A preliminary version of this paper was submitted to the XXIII International Conference on High energy physics (Berkeley, CA, July 1986).

This work was supported in part by the US Department of Energy.

\section{References}

[1] G. 't Hooft, in: Recent developments in gauge theories, Proc. NATO Advanced Study Institute (Cargese, 1979), eds. G. 't Hooft et al. (Plenum, New York, 1980).

[2] R. Rashen and H. Neuberger, Phys. Rev. Lett. 50 (1983) 1897 , and references therein.

[3] M. Veltman, Acta Phys. Pol. B 8 (1977) 475; also in: TASI lectures in elementary particle physics 1984, ed. D.N. Williams (Ann Arbor, 1984).

[4] J. Jarsak, Lattice Higgs models, Workshop on Lattice gauge theory (Wuppertal, November 1985), preprint PITHA 85/25.

[5] M.B. Einhorn, Nucl. Phys. B. 246 (1984) 75; in: TASI lectures in elementary particle physics - 1984, ed. D.N. Williams (Ann Arbor, 1984).

[6] R. Casalbuoni, D. Dominici and R. Gatto, Phys. Lett. B 147 (1984) 419.

[7] UA1 Collab., G. Arnison et a1., Phys. Lett. B 166 (1986) 484;

UA2 Collab., J.A. Appel et al., Z. Phys. C 30 (1986) 1.

[8] L. Maiani, in: TASI lectures in elementary particle physics - 1984, ed. D.N. Williams (Ann Arbor, 1984).

[9] J. Van der Bij and M. Veltman, Nucl. Phys. B 231 (1984) 205.

[10] M.B. Einhorn and H. Katsumata, in preparation.

[11] See, e.g., K. Aoki et al., Electroweak theory, Suppl. Prog. Theor. Phys. 73 (1982). 